() М.В. Шестакова

Национальный медицинский исследовательский центр эндокринологии, Москва

В статье обсуждаются результаты исследования DECLARE-TIMI 58 в аспекте ранее проведенных исследований EMPA-REG OUTCOME и CANVAS.

В рамках данных исследований все три препарата продемонстрировали возможность снижения риска госпитализации по поводу сердечной недостаточности, а также прогрессирования хронической болезни почек (ХБП). При этом популяции пациентов, включенных в исследования, отличались друг от друга. B EMPA-REG OUTCOME включались пациенты с установленным ранее диагнозом сердечно-сосудистого заболевания, в CANVAS 44,4\% пациентов не имели подтвержденного диагноза сердечно-сосудистого заболевания, но имели факторы риска развития сердечно-сосудистых осложнений, а в DECLARE TIMI 58 таких пациентов было 59,4\%.

Анализ результатов позволяет полагать, что влияние ингибиторов натрий-глюкозного котранспортера-2 (иНГлт2) на риск комбинированной сердечно-сосудистой конечной точки, включающей смерть от сердечно-сосудистой причины, инфаркт миокарда и инсульт, по всей видимости, наиболее выражено у пациентов, уже имеющих установленный диагноз сердечно-сосудистого заболевания, и это влияние достигается главным образом за счет снижения сердечно-сосудистой смертности. В популяции же пациентов с сахарным диабетом 2 типа (СД2), не имеющих установленного диагноза сердечно-сосудистого заболевания, но имеющих факторы сердечно-сосудистого риска, данный эффект не обнаруживается.

Вместе с тем снижение риска госпитализаций по поводу хронической сердечной недостаточности и замедление прогрессирования ХБП проявляется как в популяции пациентов, имеющих уже установленный диагноз сердечно-сосудистого заболевания, так и в популяции пациентов, еще не имеющих такового, но имеющих множественные факторы сердечно-сосудистого риска.

В этой связи данные, полученные в DECLARE-TIMI 58, открывают новые возможности более раннего начала терапии препаратами группы иНГЛТ-2, направленной на предупреждение и/или замедление прогрессирования нефропатии, снижение рисков появления новых случаев или усугубление имеющейся сердечной недостаточности у пациентов с СД2 вне зависимости от наличия и отсутствия у них сердечно-сосудистой патологии в анамнезе. Также объединенные данные всех трех исследований позволяют говорить об умеренном класс-эффекте иНГЛТ-2 в снижении риска атеротромботических событий у пациентов с СД2 и атеросклеротическими сердечно-сосудистыми заболеваниями в анамнезе.

КЛЮЧЕВЫЕ СЛОВА: дапаглифлозин; эмпаглифлозин; канаглифлозин

\title{
DECLARE-TIMI 58 TRIAL IN THE CONTEXT OF EMPA-REG OUTCOME AND CANVAS
}

\author{
(c) Marina V. Shestakova
}

Endocrinology Research Centre, Moscow, Russia

The article discussed results of the DECLARE-TIMI 58 study in the aspects of the previously finished trials (EMPA-REG OUTCOME and CANVAS).

All three SGLT2i demonstrated the reduction of the risk of hospitalization for heart failure, as well as the risk of progression chronic kidney disease. At the same time, the patient populations are different. In EMPA-REG OUTCOME almost all the patients had previously diagnosed cardiovascular disease; in CANVAS $44.4 \%$ of patients did not have a confirmed cardiovascular disease, but had cardiovascular risk factors, and in DECLARE TIMI 58 there were $59 \%$ of such patients.

Assessment of the published data suggests that the risk of a combined cardiovascular endpoint, including death from cardiovascular causes, myocardial infarction, and stroke, seems to be most pronounced in patients who already have established cardiovascular disease, and this effect is achieved mainly by reducing cardiovascular mortality. In the population of patients with type 2 diabetes who do not have cardiovascular disease, but who have cardiovascular risk factors, this effect is not detected. 
Contrary, risk-reduction of hospitalization for CHF and slowing the progression of CKD is manifested both in a population of patients with established cardiovascular disease and in the population of patients with multiple cardiovascular risk factors.

In this regard, DECLARE-TIMI 58, as well as previously published data, open up new option for an earlier start of SGLT-2i for primary prevention and/or slowing the progression of nephropathy, reducing the risk of heart failure, and its prevention, as well as reduction of cardiovascular morbidity and mortality in patients with type 2 diabetes, regardless of the presence or absence of cardiovascular pathology in the anamnesis.

KEYWORDS: dapagliflozin; empagliflozin; canagliflozin

В настоящее время в рутинной клинической практике продолжает оставаться актуальной проблема недостаточно ранней инициации терапии сахарного диабета 2 типа (СД2) и, как следствие, высокой частоты микро- и макрососудистых осложнений у пациентов. Наиболее значимыми осложнениями СД2 являются нефропатия и сердечно-сосудистая патология (в частности, сердечная недостаточность). Диабетическая нефропатия (ДН) является одной из наиболее частых причин развития терминальной хронической почечной недостаточности (тХПН), необходимости проведения гемодиализа или трансплантации почек: по разным оценкам, ХПН развивается у 20-40\% пациентов с СД [1], при этом ДН приводит к ТХПН в примерно 20\% случаев [2]. Сердечная недостаточность (CH) представляет собой одну из ранних и наиболее частых манифестных форм сердечно-сосудистых заболеваний при СД2, при этом для каждого $1 \%$ увеличения $\mathrm{HbA}_{1 с}$ риск развития $\mathrm{CH}$ увеличивается на $15 \%$ [3]. По данным систематического обзора 2018 г., распространенность СН среди пациентов с СД2 составляла порядка 15\% [4]. По данным регистра REACH, наличие у пациентов с СД2 $\mathrm{CH}$ увеличивало риск сердечно-сосудистой смерти примерно в 2,5 раза, а риск госпитализации по поводу $\mathrm{CH}$ - примерно в 5 раз [5]. Наличие $\mathrm{CH}$ у пациентов с СД2 сопряжено с плохим прогнозом и средней выживаемостью около 4 лет от момента постановки диагноза [6]. Это указывает на необходимость использования таких схем лечения, которые предотвращают прогрессирование сердечно-сосудистых заболеваний, включая СН у пациентов С СД2.

DECLARE-TIMI 58 - исследование, проведенное с целью оценки влияния селективного ингибитора натрий-глюкозного котранспортера 2 типа (иНГЛТ-2) дапаглифлозина (ДАПА) на кардиоваскулярные и ренальные исходы у пациентов с СД2, имевших атеросклеротические сердечно-сосудистые заболевания (АССЗ) или множественные факторы риска их развития [7-9].

\section{DECLARE-TIMI 58}

\section{Материал и методы}

DECLARE-TIMI 58 - мультинациональное проспективное рандомизированное двойное-слепое плацебо-контролируемое исследование III фазы у пациентов с СД2 и установленными АССЗ или множественными факторами риска их развития. Исследование было разработано и реализовано группой TIMI (Thrombolysis in Myocardial Infarction (TIMI) Study Group, США), медицинской opганизацией «Хадасса» (Hadassah Medical Organization, Израиль) и исполнительным комитетом исследования. Спонсором выступила компания AstraZeneca. В исследо- вании приняли участие 882 исследовательских центра из 33 стран, включая Россию [7-9].

Исходный дизайн исследования учитывал одну первичную конечную точку - развитие у пациентов крупных сердечно-сосудистых событий (major adverse cardiovascular events, MACE) [7]. Однако он был изменен [10] в связи с обнародованными результатами исследования EMPA-REG OUTCOME [11] (в котором оценивался другой иНГЛТ-2, эмпаглифлозин), согласно которому применение эмпаглифлозина в большей степени было связано с уменьшением случаев сердечно-сосудистой смерти и случаев госпитализации по поводу $\mathrm{CH}$, чем с влиянием на МАСЕ. В этой связи исполнительный комитет исследования внес поправки в протокол и, наряду с МАСЕ, выделил вторую первичную конечную точку: комбинированный показатель случаев сердечно-сосудистой смерти или госпитализации по поводу СН (появление новых случаев $\mathrm{CH}$ или усугубление имеющейся $\mathrm{CH}$, которые требовали госпитализации). Важно подчеркнуть, что к моменту принятия этого решения отсутствовали какие-либо промежуточные результаты по развитию MACE у пациентов в рамках исследования DECLARE-TIMI 58 [8].

В исследование включались пациенты с СД2, имевшие уровень гликогемоглобина $\mathrm{A} 1 \mathrm{c}\left(\mathrm{HbA}_{1 \mathrm{c}}\right)$ 6,5-12\% и клиренс креатинина не менее 60 мл/мин по формуле Кокрофта-Голта. Пациенты должны были иметь подтвержденное ACC3 (ишемическая болезнь сердца, ишемическая цереброваскулярная болезнь или болезнь периферических артерий) или множественные факторы риска его развития. При наличии в анамнезе подтвержденного АСС3 минимальный возраст пациентов составлял 40 лет, независимо от пола. Условием включения пациентов с множественными факторами риска был возраст (для мужчин 55 лет и старше, для женщин - 60 лет и старше) и наличие как минимум 1 традиционного фактора риска развития АССЗ, включая артериальную гипертензию, дислипидемию (ЛПНП >130 мг/дл или 3,36 ммоль/л или факт применения гиполипидемической терапии) и курение табака $[8,9]$.

Скринированные пациенты включались в слепой вводный период исследования (4-8 нед), на протяжении которого все они получали плацебо и должны были являться в исследовательский центр для забора крови и мочи с целью лабораторного анализа. По окончании вводного периода субъекты, соблюдавшие процедуры исследования, были рандомизированы в 2 группы (в соотношении 1:1): 1-я группа - пациенты, получавшие ДАПА ежедневно в дозе 10 мг 1 раз в день; 2-я группа - пациенты, получавшие плацебо. Во время исследования применение иНГЛТ-2, за исключением ДАПА, было запрещено. Использование сахароснижающих препаратов из других групп оставалось на усмотрение лечащего врача. Визи- 
ты пациентов в исследовательские центры проводились 1 раз в 6 мес. На каждом из них осуществлялись забор крови и мочи для лабораторного анализа, оценка нежелательных явлений и оценка приверженности терапии. Также с интервалом 3 мес между визитами осуществлялся контакт пациента с исследователями посредством телефонного звонка $[8,9]$.

Как было уже упомянуто, в исследовании оценивались две первичные конечные точки эффективности: комбинированный показатель случаев сердечно-сосудистой смерти или госпитализации по поводу $\mathrm{CH}$, а также частота МАCЕ. Вторичные конечные точки включали комбинированную почечную конечную точку, а также случаи смерти от любых причин. Почечная конечная точка включала частоту развития устойчивого снижения (на 40\% и более) расчетной скорости клубочковой фильтрации (рСКФ) ниже 60 мл/ мин, развитие терминальной стадии почечной недостаточности или смерть от почечной либо сердечно-сосудистой причины. Для оценки эффекта исследуемого препарата только на почечные события дополнительно оценивалась почечная конечная точка в «чистом виде», которая соответствовала комбинированной почечной конечной точке, за исключением смерти от сердечно-сосудистых причин. Кроме того, комитетом по мониторингу клинических событий группы TIMI осуществлялся дополнительный анализ отдельных компонентов первичных и вторичных конечных точек эффективности у исследуемых пациентов. Отдельно анализировались все серьезные нежелательные явле-

Таблица 1. Исходные характеристики пациентов ния (СНЯ) и все нежелательные явления (НЯ), повлекшие за собой выбытие пациентов из исследования [8, 9].

Первичная оценка сердечно-сосудистой безопасности и эффективности была проведена на основании данных, полученных от 17160 рандомизированных пациентов. В расчет принимались пациенты, получившие исследуемый препарат хотя бы один раз. Дополнительно анализ основных конечных точек исследования был стратифицирован по наличию АССЗ (пациенты с установленным АСС3 и пациенты с множественными факторами развития $A C(3)[8,9]$.

\section{РЕЗУЛЬТАТЫ}

В исследование были рандомизированы 17160 человек, при этом меньшая часть пациентов - 6974 пациента (40,6\%) представляла собой участников с установленными АСС3, а большая часть - 10168 пациентов (59,4\%) с множественными факторами риска развития АСС3 [8].

Группы пациентов были сопоставимы между собой по исходным характеристикам (табл. 1). Средний уровень

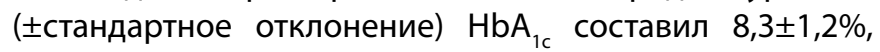
со средней продолжительностью СД2 - 11 лет (межквартильный размах 6,0-16,0 лет). Средняя рСКФ составила 85,2 мл/мин/1,73 м²: 45\% пациентов исходно имели рСКФ в интервале 60-90 мл/мин/1,73 м², 7\% пациентов - менее 60 мл/мин/1,73 м² и 48\% пациентов - выше 90 мл/ мин/ 1,73 м² На момент включения в исследование 10\% пациентов имели в анамнезе $\mathrm{CH}$ [8].

\begin{tabular}{|c|c|c|}
\hline Характеристика & $\begin{array}{l}\text { Дапаглифлозин } \\
\text { (N = 8582) }\end{array}$ & $\begin{array}{l}\text { Плацебо } \\
(\mathbf{N}=\mathbf{8 5 7 8})\end{array}$ \\
\hline Возраст, годы & $63,9 \pm 6,8$ & $64,0 \pm 6,8$ \\
\hline Женский пол, n (\%) & $3171(36,9)$ & $3251(37,9)$ \\
\hline Длительность СД2, годы & $11,0(6,0-16,0)$ & $10,0(6,0-16,0)$ \\
\hline Гликированный гемоглобин, \% & $8,3 \pm 1,2$ & $8,3 \pm 3,2$ \\
\hline рСКФ (мл/мин/1,73 м²) & $85,4 \pm 15,8$ & $85,1 \pm 16,0$ \\
\hline $\begin{array}{l}\text { Наличие атеросклеротического сердечно-сосудистого } \\
\text { заболевания, n (\%) }\end{array}$ & $3474(40,5)$ & $3500(40,8)$ \\
\hline ИБС в анамнезе, n (\%) & $2824(32,9)$ & $2834(33,0)$ \\
\hline Заболевание периферических артерий в анамнезе, n (\%) & $522(6,1)$ & $503(5,9)$ \\
\hline Цереброваскулярные заболевания в анамнезе, n (\%) & $653(7,6)$ & $648(7,6)$ \\
\hline Сердечная недостаточность в анамнезе, n (\%) & $852(9,9)$ & $872(10,2)$ \\
\hline \multicolumn{3}{|l|}{ Сопутствующая сердечно-сосудистая терапия, n (\%): } \\
\hline антиагреганты & $5245(61,1)$ & $5242(61,1)$ \\
\hline ингибиторы АПФ или блокаторы рецепторов ангиотензина II & $6977(81,3)$ & $6973(81,3)$ \\
\hline 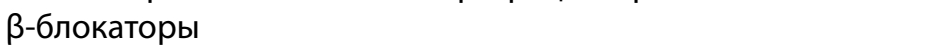 & $4498(52,4)$ & $4532(52,8)$ \\
\hline статины или эзетимиб & $6432(74,9)$ & $6436(75,0)$ \\
\hline диуретики & $3488(40,6)$ & $3479(40,6)$ \\
\hline
\end{tabular}

Примечание. В таблице для средних показателей после знака \pm приведено стандартное отклонение; $\mathrm{n}-$ абсолютное количество пациентов; АД - артериальное давление; СКФ - скорость клубочковой фильтрации; рСКФ - расчетная скорость клубочковой фильтрации; ИБС - ишемическая болезнь сердца; АПФ - ангиотензинпревращающий фермент. 


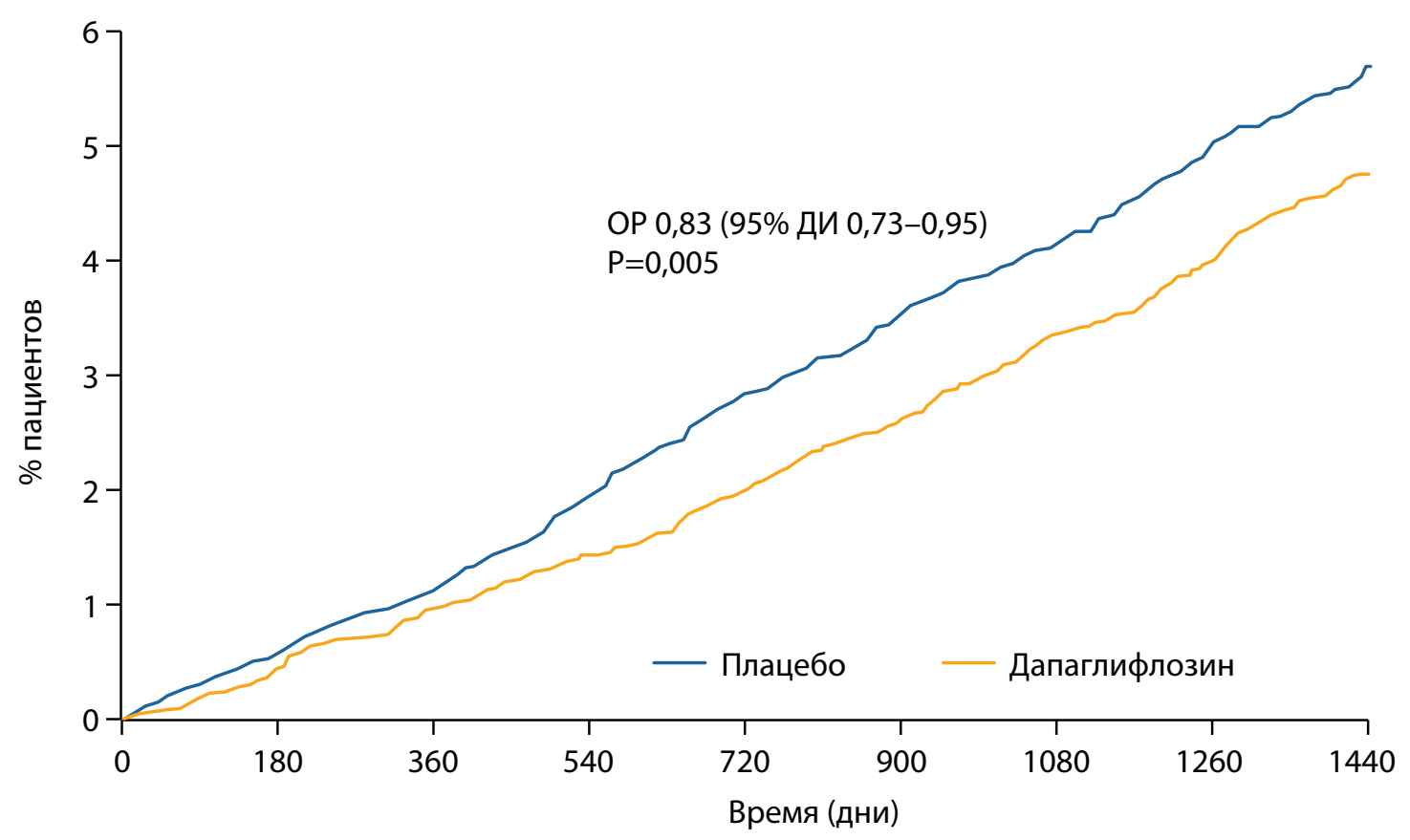

Рис. 1. Частота возникновения первичной конечной точки «сердечно-сосудистая смерть или госпитализация по поводу сердечной недостаточности» (адаптировано по [8]). ОР - относительный риск; ДИ - доверительный интервал

Первичная конечная точка «сердечно-сосудистая

смертность или госпитализация по поводу СН»

у пациентов, получавших ДАПА, совокупная частота возникновения случаев сердечно-сосудистой смерти и госпитализации по поводу СН была значимо ниже (4,9\%) по сравнению с пациентами, получавшими плацебо (5,8\%) (ОР 0,83; 95\% ДИ 0,73-0,95; p=0,005). Таким образом, относительное снижение рисков наступления данной конечной точки составило 17\% (рис. 1).

При этом у пациентов, получавших ДАПА, основным «драйвером» наблюдаемого снижения частоты развития событий данной комбинированной точки явилось значимое снижение на $27 \%$ риска развития госпитализаций по поводу СН (ОР 0,73; 95\% ДИ 0,61-0,88). Различий в частоте сердечно-сосудистой смерти в группах сравнения в общей популяции выявлено не было (ОР 0,98; 95\% ДИ 0,82-1,17) [8].

Эффективность ДАПА в отношении комбинированного показателя случаев сердечно-сосудистой смерти или госпитализации по поводу СН была сопоставимой между подгруппой пациентов с установленными АСС3 (7,8\% пациентов, получавших ДАПА, и 9,3\% пациентов, получавших плацебо; ОР 0,83; 95\% ДИ 0,71-0,98) и подгруппой пациентов с множественными факторами риска развития АСС3 (2,8\% пациентов, получавших ДАПА, и 3,4\% пациентов, получавших плацебо; ОР 0,84; $95 \%$ ДИ 0,67-1,04) (рис. 2). При этом различия между исследуемым препаратом и плацебо в первом случае достигли статистически значимых различий, а во втором случае - нет [8]. Статистический анализ данных исследования проводился с учетом принципа иерархического анализа конечных точек, то есть, если по первичной конечной точке была показана достоверность различий, то показатель р для вторичных конечных точек не рассчитывался. В этой связи, ниже достоверность различий указана только для тех показателей, которые есть в первоисточнике. Для оценки достоверности различий и разброса данных следует ориентироваться на показатель ДИ.

\section{Первичная конечная точка «МАCE»}

По результатам анализа другой первичной конечной точки - МАСЕ - ДАПА соответствовал критерию «не меньшей эффективности» при сравнении с плацебо (верхняя граница 95\% ДИ <1,3; p<0,001 для критерия «не меньшей эффективности») (рис. 3) [8].

Несмотря на то что у пациентов, получавших ДАПА, случаи МАСЕ регистрировались номинально реже $(8,8 \%)$ по сравнению с пациентами, получавшими плацебо (9,4\%), статистически значимого различия в общей популяции исследования по данному показателю между группами получено не было (ОР 0,93; 95\% ДИ 0,84-1,03; $\mathrm{p}=0,17)[8]$.

Эффективность ДАПА в отношении случаев МАСЕ также была сопоставимой между подгруппой пациентов с установленными АСС3 (13,9\% пациентов, получавших ДАПА, и 15,3\% пациентов, получавших плацебо; ОР 0,90; 95\% ДИ 0,79-1,02) и подгруппой пациентов с множественными факторами риска развития АСС3 (5,3\% пациентов, получавших ДАПА, и 5,2\% пациентов, получавших плацебо; ОР 1,01; 95\% ДИ 0,86-1,20) [8].

Анализ дополнительных конечных точек

У пациентов, получавших ДАПА, частота случаев почечной конечной точки (устойчивое снижение рСКФ на $40 \%$ и более, развитие терминальной стадии почечной недостаточности или смерть от почечной либо сердечно-сосудистой причины) составила 4,3\% у пациентов, получавших ДАПА, и 5,6\% в группе плацебо (ОР 0,76; 95\% ДИ 0,67-0,87), что представляет собой снижение относительного риска на 24\% (рис. 4). 


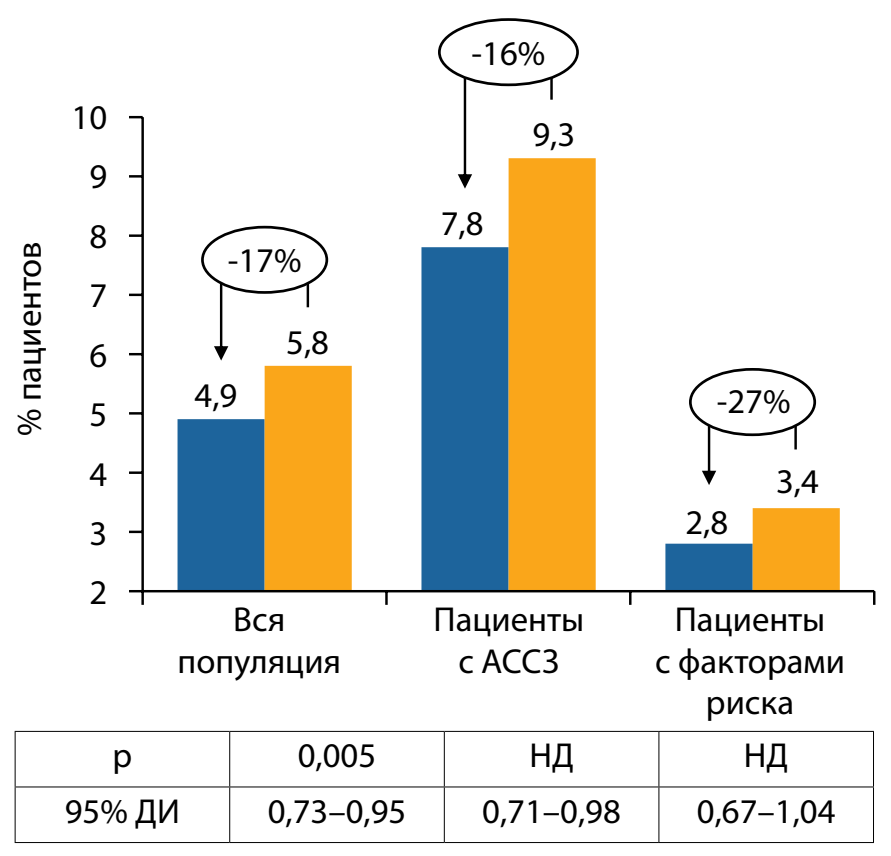

* НД - показатель не рассчитывался

Рис. 2. Частота возникновения первичной конечной точки «сердечнососудистая смерть или госпитализация по поводу сердечной недостаточности в различных популяциях».

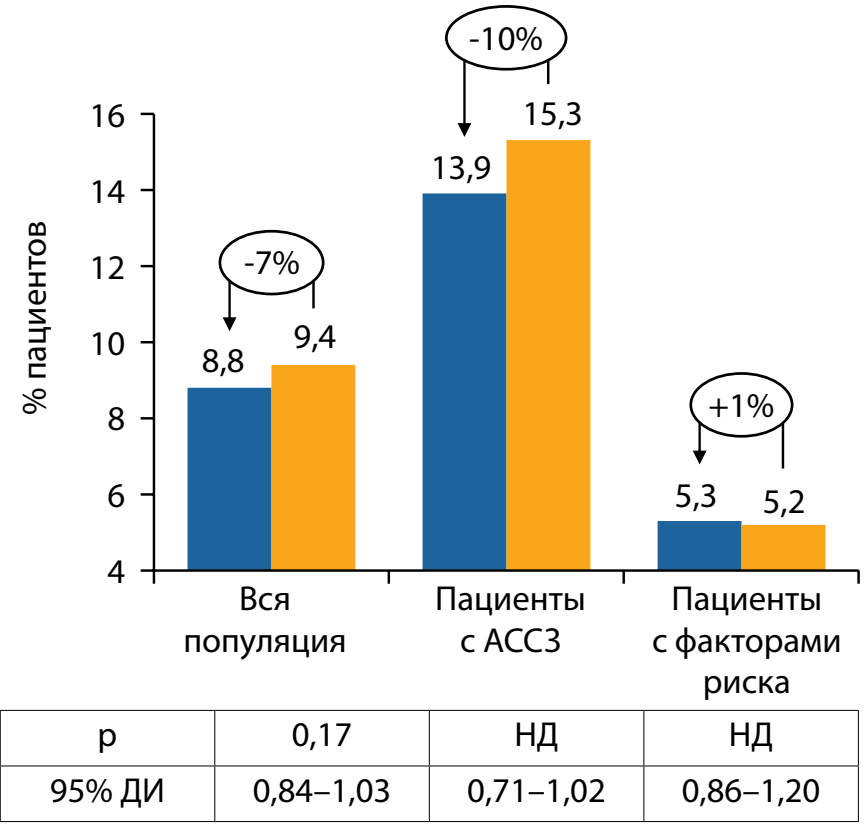

Дапаглифлозин

Плацебо

Рис. 3. Частота возникновения больших сердечно-сосудистых событий (MACE) в различных популяциях.

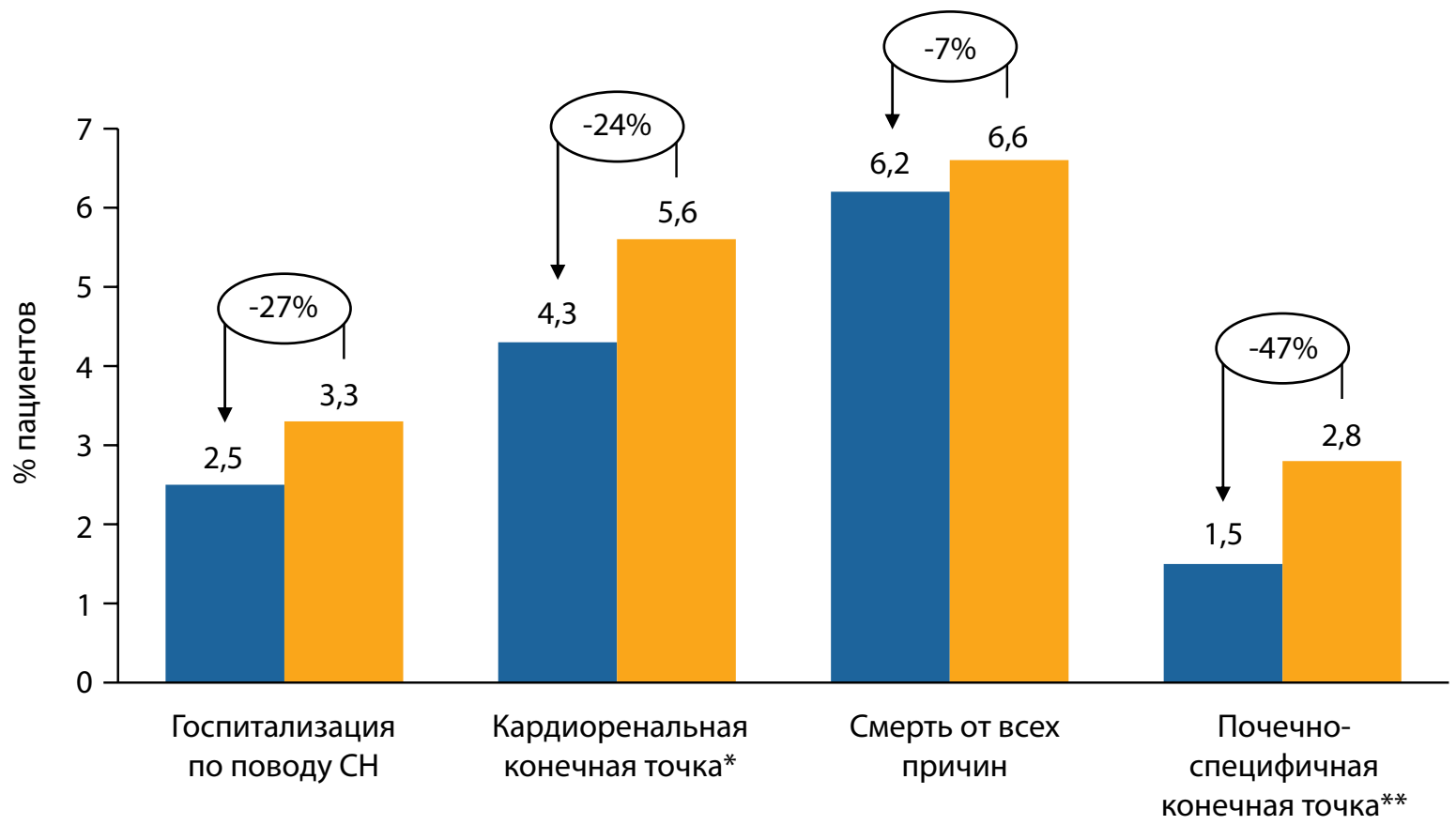

\begin{tabular}{|c|c|c|c|c|}
\hline $\mathrm{p}$ & НД & $\mathrm{p}<0,0001$ & НД & $\mathrm{p}<0,0001$ \\
\hline $95 \%$ ДИ & $0,84-1,03$ & $0,67-0,87$ & $0,82-1,04$ & $0,43-0,66$ \\
\hline
\end{tabular}

Рис. 4. Частота возникновения вторичных конечных точек в исследовании DECLARE.

* Устойчивое снижение (на 40\% и более) расчетной скорости клубочковой фильтрации (рСКФ), развитие терминальной стадии почечной недостаточности или смерть от почечной либо сердечно-сосудистой причины).

** Почечно-специфичная конечная точка - кардиоренальная конечная точка без смерти от сердечно-сосудистых причин. 
Следует отметить, что эти преимущества применения ДАПА наблюдались независимо от наличия или отсутствия установленного АССЗ или от степени исходного снижения почечной функции, как это было показано в недавнем субанализе данных по почечным исходам, опубликованном в журнале Lancet в 2019 г. [12]. 221 пациент со снижением рСКФ 40\% и более от исходного значения получали плацебо, и лишь 120 пациентов - ДАПА, что соответствует 46\% снижению риска ухудшения почечной функции (ОШ 0,54; $\mathrm{P}<0,0001$ ). Аналогичный показатель в отношении снижения рисков ТХПН или смерти от «почечных причин» составил 59\% (OP 0,41; $\mathrm{P}=0,012$ ).

Важно отметить, что на этапе скрининга лишь небольшая доля пациентов имела выраженное снижение почечной функции. Столь значимые результаты, продемонстрированные у пациентов с относительно сохранной фильтрационной функцией почек, являются подтверждением тезиса о том, что применение препарата с целью профилактики почечной недостаточности оправдано не только среди пациентов со скомпрометированной функцией почек, но и в качестве первичной профилактики почечных осложнений.

Частота случаев смерти от любых причин не имела статистически значимых различий между группами, хотя в числовых значениях и была ниже в группе ДАПА (6,2\% в группе ДАПА и 6,6\% в группе плацебо; ОР 0,93; 95\% ДИ $0,82-1,04)[8]$.

\section{Анализ безопасности}

В ходе исследования также оценивались дополнительные параметры безопасности. Так, в группе ДАПА была зарегистрирована достоверно меньшая частота СНЯ - 34,1\%, в то время как в группе плацебо - у 36,2\% пациентов (ОР 0,91; 95\% ДИ 0,87-0,96; p<0,001).

В группе ДАПА частота встречаемости тяжелой гипогликемии была значимо ниже $(0,7 \%)$ по сравнению с группой плацебо (1,0\%) (ОР 0,68; 95\% ДИ 0,49-0,95; $\mathrm{p}=0,02)$. Аналогично, при применении ДАПА частота Встречаемости острого повреждения почек (1,5\%) и рака мочевого пузыря (0,3\%) была значимо ниже по сравнению с применением плацебо (2,0\% и 0,5\% соответственно) (для острого повреждения почек: ОР 0,69; 95\% ДИ 0,55-0,87; $p=0,002 ;$ для рака мочевого пузыря: ОР 0,57; 95\% ДИ 0,35-0,93; $p=0,02)$.

Частота встречаемости ампутаций, переломов, гиповолемии и реакций гиперчувствительности была сопоставима между группами. Частота развития диабетического кетоацидоза была значимо выше (0,3\%) у пациентов, получавших ДАПА, по сравнению с пациентами группы плацебо (0,1\%) (ОР 2,18; 95\% ДИ 1,10-4,30; $p=0,02)$, при этом абсолютное число данных нежелательных явлений было невелико (ДАПА/плацебо, соответственно 27/12). Следует отметить, что более $80 \%$ пациентов с диабетическим кетоацидозом исходно получали инсулин.

Генитальные инфекции, которые привели к выбытию из исследования или рассматривались как СНЯ, чаще встречались у пациентов, получавших ДАПА (0,9\%), по сравнению с пациентами, получавшими плацебо $(0,1 \%)$, вне зависимости от пола пациентов (ОР 8,36; 95\% ДИ 4,19-16,68; p<0,001). Необходимо отметить, что данное НЯ, расцененное исследователями как серьезное, в исследовании встречалось редко (по 2 события в каждой группе) [8].

\section{ОБСУЖДЕНИЕ}

\section{Исследования сердечно-сосудистой безопасности} препаратов класса ингЛТ-2

DECLARE-TIMI 58 является третьим исследованием, целью которого было изучение влияния иНГЛТ-2 на развитие сердечно-сосудистых осложнений у пациентов с СД2. В проспективное рандомизированное исследование EMPA-REG OUTCOME [13] были включены 7020 пациентов с СД2 и установленным АСС3, которые получали эмпаглифлозин или плацебо в дополнение к стандартной сахароснижающей терапии. По результатам исследования применение эмпаглифлозина привело к снижению риска развития МАCE на 14\% по сравнению с плацебо (ОР 0,86; 95\% ДИ 0,74-0,99; $p<0,001$ для критерия «не меньшей эффективности»; $p=0,04$ для критерия «превосходства»). Данное различие достигалось в первую очередь за счет снижения случаев сердечно-сосудистой смерти (ОР 0,62; 95\% ДИ 0,49-0,77; $\mathrm{p}<0,001)$. Применение эмпаглифлозина было также ассоциировано со снижением риска госпитализации по поводу СН (ОР 0,65; 95\% ДИ 0,50-0,85) и снижением риска развития или ухудшения существующей нефропатии (ОР 0,61; 95\% ДИ 0,53-0,70) [11, 14].

B аналогичную исследовательскую программу CANVAS (исследования CANVAS и CANVAS-R) [15] были включены 10142 пациента с СД2, имевшие установленное ACC3 (65,6\%) или факторы риска его развития (34,4\%), которые получали канаглифлозин либо плацебо в дополнение к стандартной сахароснижающей терапии. Результаты исследования продемонстрировали, что применение канаглифлозина сопровождается снижением риска развития МАСЕ на 14\% по сравнению с плацебо (ОР 0,86; 95\% ДИ 0,75-0,97; $\mathrm{p}<0,001$ для критерия «не меньшей эффективности»; $p=0,02$ для критерия «превосходства»). При этом статистически значимых различий по отдельным компонентам МАCЕ (сердечно-сосудистая смерть, нефатальный инфаркт миокарда или инсульт) между группой канаглифлозина и группой плацебо получено не было [15].

Также применение канаглифлозина было ассоциировано с возможным снижением риска госпитализации по поводу СН (ОР 0,67; 95\% ДИ 0,52-0,87) и возможным снижением риска развития ренальных осложнений (прогрессирование альбуминурии: ОР 0,73; 95\% ДИ 0,67-0,79; комбинированный показатель случаев устойчивого снижения рСКФ $\geq 40 \%$, заместительной почечной терапии или смерти от почечных причин: ОР 0,60; 95\% ДИ 0,47-0,77) [15].

Различия в популяциях исследований DECLARE-TIMI 58, EMPA-REG OUTCOME и CANVAS

Следует отметить, что популяции пациентов DECLARETIMI 58, EMPA-REG OUTCOME и CANVAS существенно отличались друг от друга в первую очередь по наличию сопутствующих АСС3 и их тяжести (рис. 5) [8, 11, 15].

В исследование EMPA-REG OUTCOME включались только пациенты с уже установленными сердечно-сосудистыми заболеваниями, в то время как в исследования 


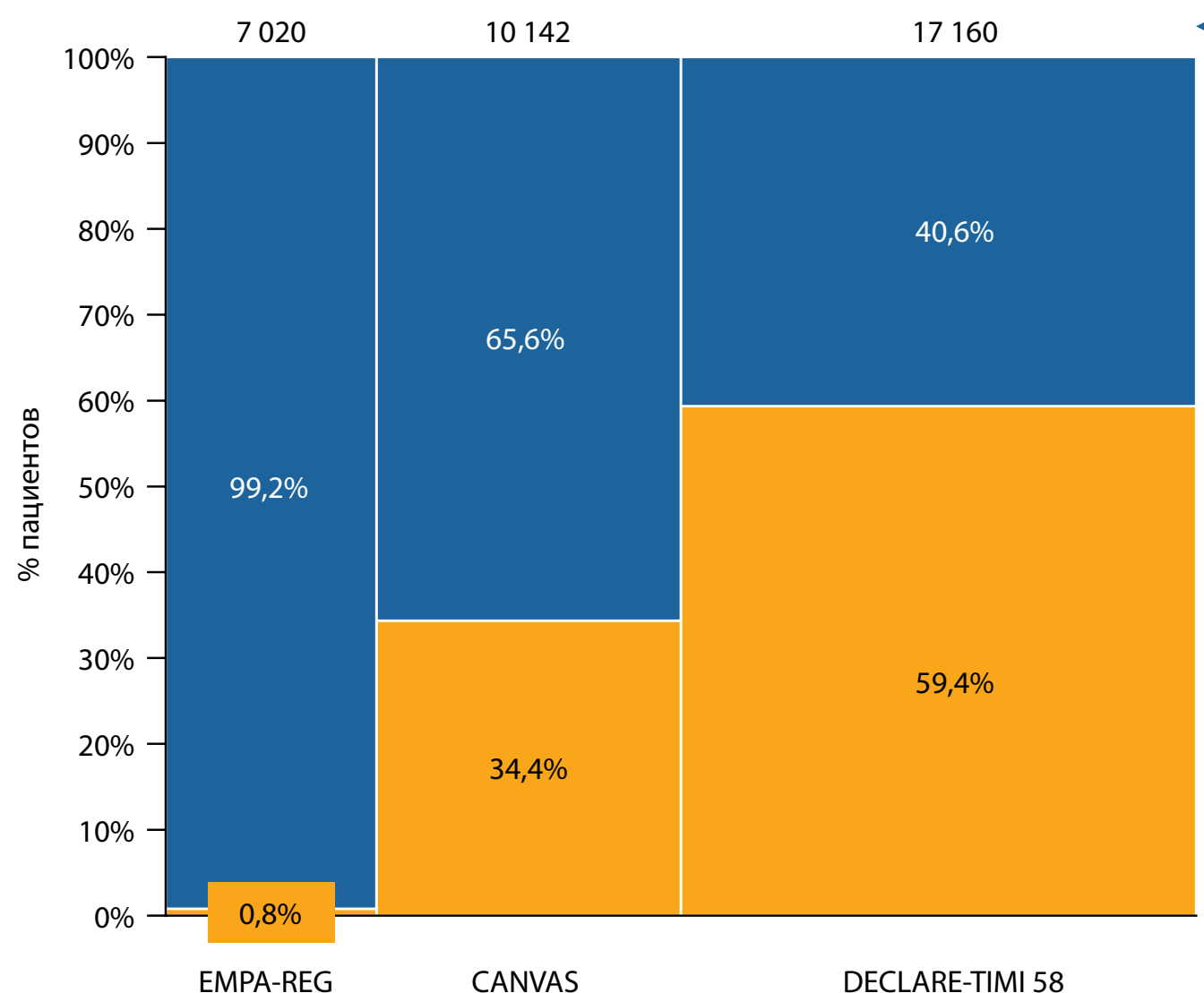

CANVAS
DECLARE-TIMI 58

\section{Кол-во пациентов в исследовании}

Пациенты с установленными АСС3

Пациенты с факторами риска развития АСС3

Рис. 5. Соотношение пациентов с установленными атеросклеротическими сердечно-сосудистыми заболеваниями и пациентов с факторами риска развития атеросклеротических сердечно-сосудистых заболеваний в исследованиях EMPA-REG OUTCOME, CANVAS и DECLARE-TIMI 58.

DECLARE-TIMI 58 и CANVAS также включались пациенты с факторами риска их развития. При этом если в исследовании CANVAS доля пациентов без установленного диагноза сердечно-сосудистого заболевания составляла 34,4\%, то в исследовании DECLARE-TIMI 58 их доля составила почти $60 \%$.

Следует особо отметить, что пациенты различались и по средней СКФ (в DECLARE-TIMI 58 средняя СКФ составила 85,2 мл/мин/1,72 м² в сравнении с 74,1 в EMPA-REG и 76,5 - в исследовании (ANVAS).

Также между исследованиями можно обнаружить и ряд других различий в характеристиках пациентов (по полу, наличию сопутствующих заболеваний и др.). Все эти различия позволяют объяснить расхождения в полученных результатах $[8,11,15]$.

О6 имеющейся разнице исходной тяжести и степени сердечно-сосудистого (СС) риска между пациентами в трех исследованиях свидетельствует и наблюдаемая частота СС-смерти в группе плацебо, которая значительно варьирует, различаясь между исследованиями в 2 раза. Исходя из вышесказанного можно заключить, что для выявления четких препарат-специфичных различий необходимы дополнительные исследования, направленные на прямое сравнение препаратов иНГЛТ2 друг с другом.
Метаанализ исследований DECLARE-TIMI 58, EMPA-REG

\section{OUTCOME и CANVAS}

Zelniker T. и соавт. [16] провели метаанализ результатов исследований DECLARE-TIMI 58, EMPA-REG OUTCOME и CANVAS (общая популяция 34322 человек) с целью оценки влияния иНГЛТ2 на кардиоваскулярные и ренальные исходы у пациентов с СД2 (рис. 6). Было установлено, что наиболее значимым эффектом применения иНГЛТ2 являлось снижение риска госпитализации по поводу СН (на $31 \%$ ) и прогрессирования почечной недостаточности (на 45\%).

Влияние иНГЛТ2 на снижение риска MACE было умеренным (на 11\%), но все же значимым. В то же время авторы подчеркнули, что в различных исследованиях эффект иНГЛТ2 на риск развития атеротромботических событий (MACE) зависел от исследуемой популяции. Уменьшение частоты МАСЕ было статистически значимым только у пациентов с установленными АСС3 [16]. Это коррелирует с данными, согласно которым частота случаев госпитализаций по поводу СН в группах плацебо составляла около 14 на 1000 пациенто-лет среди пациентов с установленными АСС3 и около 4-5 на 1000 пациенто-лет среди пациентов только с множественными факторами риска развития АСС3 [17]. 


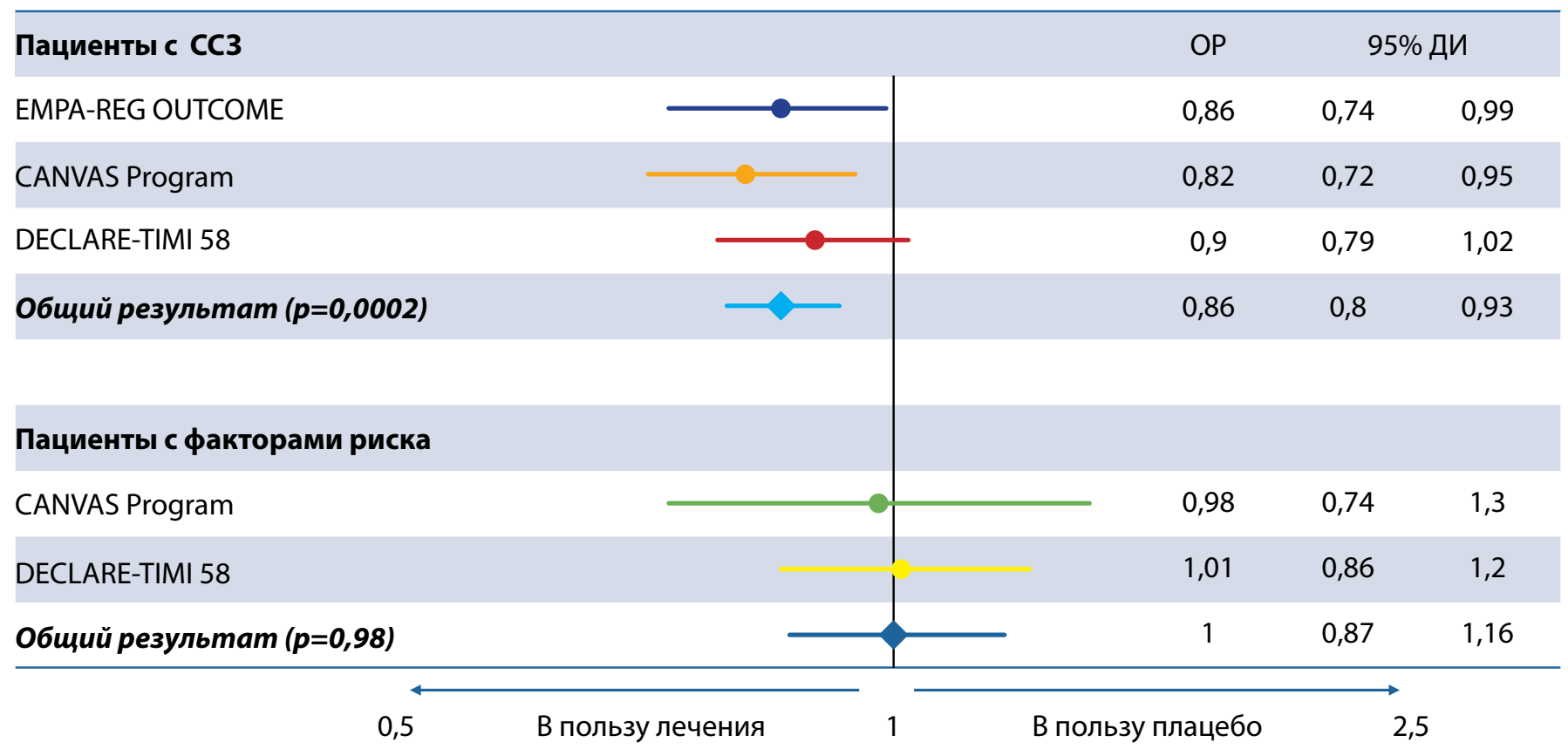

Рис. 6. Метаанализ основной комбинированной конечной точки (MACE) в исследованиях DECLARE-TIMI 58, EMPA-REG OUTCOME и CANVAS(адаптировано по [16]).

В то же время влияние иНГЛТ2 на частоту госпитализаций по поводу СН и частоту прогрессирования почечной недостаточности не зависело от наличия у пациентов АСС3. При этом наблюдалась прямая зависимость между исходной фильтрационной функцией почек у пациентов с СД2 и клиническим эффектом иНГЛТ2: максимальный нефропротективный эффект наблюдался в группе пациентов с рСКФ более 90 мл/мин - снижение относительного риска на 56\%; при рСКФ 60-90 данное снижение составляло порядка 44\% и было еще менее выраженным при рСКФ менее 60 мл/мин - 33\%.

Противоположная корреляция наблюдалась для $\mathrm{CH}$ : максимальное снижение риска $\mathrm{CH}$ на 40\% наблюдалось в группе пациентов с наиболее низкой рСКФ; на $31 \%$ в группе с пациентов с рСКФ 60-90 мл/ мин; влияние на риск СН было статистически не значимым в группе с рСКФ 90 мл/мин и более - 12\% [16].

В целом иНГЛт2 имели хорошую переносимость и приемлемый профиль безопасности. При этом стоит отметить, что при применении иНГЛТ2 у пациентов был повышен риск возникновения микотической урогенитальной инфекции, которая обычно легко подвергалась лечению и редко рецидивировала [16].

Результаты оценки риска развития нежелательных явлений, проведенной в упомянутом метаанализе [16], показывают, что применение иНГЛТ2, вероятно, ассоциировано с повышенным риском диабетического кетоацидоза. В то же время частота развития диабетического кетоацидоза была очень низкой во всех трех исследованиях, и риск его развития может быть уменьшен в случае, если пациенты будут осведомлены о проблеме и насторожены. Данные о возможной связи использования иНГЛТ2 с развитием инсульта не нашли подтверждения. Повышенный риск ампутаций и переломов наблюдался только в одном исследовании (CANVAS) [15].

В результате проведенного метаанализа [16] было показано, что иНГЛт2 могут применяться у пациентов с СД2 вне зависимости от наличия АССЗ и СН в анамнезе, так как они безопасно снижают уровень $\mathrm{HbA}_{1 c^{\prime}}$ а также риск госпитализации по поводу СН и прогрессирования почечной недостаточности у широкого круга пациентов $[8,11,15]$.

На основании полученных данных можно сделать вывод о том, что снижение риска госпитализации по поводу СН и прогрессирования почечной недостаточности следует ожидать у всех пациентов с СД2. В то же время снижение риска развития МАСЕ и сердечно-сосудистой смерти при применении иНГЛТ2 будет выраженным только у пациентов с установленными АСС3. Дополнительные данные о потенциале инГЛТ2 в отношении влияния на риск прогрессирования СН или ХБП у пациентов без СД2 будут предоставлены профессиональному сообществу в ближайшее время, когда станут известны результаты ряда исследований, направленных на изучение этих вопросов.

Несмотря на пристальное изучение, механизм действия иНГЛт2 пока остается малоизученным [18]. На сегодняшний день существует мнение, что контроль гликемии у пациентов с СД2 приводит к снижению микрососудистых, но не макрососудистых осложнений [19]. Имеются данные, что уменьшение частоты госпитализаций по поводу СН при применении иНГЛт2 может быть связано с нефропротективным эффектом и натрийурезом, которые они вызывают [16, 18, 20]. Замедление прогрессирования почечной недостаточности и снижение частоты госпитализаций по поводу $\mathrm{CH}$, а также уменьшение количества и объема сопутствующих вмешательств (по поводу $\mathrm{CH}$ и почечной недостаточности) и ассоциированных с ними осложнений могут влиять на снижение риска как сердечно-сосудистой смерти, так и смерти от любых причин [16].

Дапаглифлозин и первичная профилактика сердечно-

сосудистых осложнений у пациентов с СД2

Результаты исследования DECLARE-TIMI 58 продемонстрировали преимущества ДАПА в сравнении с плацебо 
в снижении риска развития сердечно-сосудистых осложнений (хронической СН) и ХБП в широкой популяции пациентов с СД2 [8].

Результаты предыдущих двух исследований сердечно-сосудистых исходов при применении иНГЛТ-2 (ЕМРАREG OUTCOME и CANVAS) давали понимание влияния глифлозинов на сердечно-сосудистые риски преимущественно у пациентов с уже развившимся АССЗ.

Полученные результаты крайне важны для обоснования применения у пациентов с СД2 как ДАПА, так и класса препаратов иНГЛТ2 в целом с целью «первичной» профилактики сердечно-сосудистых осложнений.

Необходимо отметить, что по сравнению с другими иНГЛT-2 в DECLARE-TIMI 58 ДАПА только номинально (без статистически значимой разницы между группами) снижал частоту развития событий конечной точки MACE в общей популяции пациентов. Это может объясняться принципиальными различиями популяции данного исследования в сравнении с теми, кто включался в два других CVOT-исследования: в частности, в DECLARE-TIMI 58 пациенты характеризовались исходно более низким сердечно-сосудистым риском [7, 9, 11, 15, 21]. Однако эффективность ДАПА в отношении риска сердечно-сосудистых событий была показана в популяции пациентов, имеющих ИМ в анамнезе [10]. В данной подгруппе пациентов ( $n=3584)$ снижение риска развития событий первичной комбинированной конечной точки (MACE) было значимым и составило 16\% (ОР 0,84; 95\% ДИ 0,72-0,99; $\mathrm{p}=0,039)$ [10]. Также было показано, что в данной когорте пациентов терапия ДАПА сопровождалась снижением риска развития событий комбинированной конечной точки, включавшей СН или СС-смерть, на 19\% (OP 0,81; 95\% ДИ 0,65-1,00; $\mathrm{p}=0,046)$.

Не менее значимы и интересны результаты предварительно запланированного подгруппового анализа DECLARE-TIMI 58, показавшего влияние ДАПА на снижение риска СС- и общей смертности в подгруппе пациентов с СН со сниженной фракцией выброса левого желудочка ( $\mathrm{n}=671$, или 3,9\% от общей популяции исследования). Терапия ДАПА на протяжении 4-летнего периода приводила к выраженному снижению риска возникновения смерти от СС-причин на 45\% (ОР 0,55; 95\% ДИ 0,34-0,90) и риска смерти от всех причин на $41 \%$ (ОР 0,59; 95\% ДИ 0,40-0,88) [22]. В этой когорте пациентов, в группе ДАПА, было продемонстрировано снижение частоты событий комбинированного показателя СС-смерть/ госпитализация по поводу СН на 38\% (ОР 0,62; 95\% ДИ 0,45-0,86).

Продемонстрированный в DECLARE-TIMI 58 значимый нефропротективный эффект в первую очередь у пациентов с исходно сохраненной функцией почек дает основание для стратегии более раннего назначения ДАПА с целью предупреждения или предотвращения прогрессирования нефропатии, то есть с целью первичной профилактики [8].

\section{Вопросы безопасности}

Клиническая значимость результатов исследования DECLARE-TIMI 58 заключается, в том числе, в подтверждении профиля безопасности длительного использования ДАПА при СД2 с точки зрения риска развития таких осложнений, как рак молочной железы и мочевого пу- зыря, ампутации, переломы, гангрена Фурнье и острое повреждение почек.

\section{ЗАКЛЮЧЕНИЕ}

Агрегированные данные последних CVOT-исследований [16] позволяют сделать вывод о том, что иНГЛТ-2 обладают умеренным эффектом в отношении МАСЕ, ограниченным популяцией пациентов с установленным диагнозом АСС3. Тем не менее применение ингЛТ-2, в частности ДАПА, сопровождалось убедительными эффектами снижения частоты госпитализаций по поводу $\mathrm{CH}$ и прогрессирования болезни почек независимо от наличия АCС3 или $\mathrm{CH}$.

В марте 2019 г. в рекомендации АCC/AНА по первичной профилактике ССЗ были внесены важные изменения. Указывается, что, несмотря на то, что имеются лишь ограниченные данные о применении препаратов группы иНГЛТ-2 у пациентов высокого риска, их использование с целью первичной профилактики СС3 может принести пациентам преимущества и может быть рассмотрено у пациентов с СД2, $\mathrm{HbA}_{1 c} \geq 7,0 \%$ и наличием факторов риска СС3 [23]. Аналогичные изменения были внесены в обновленные Российские рекомендации по лечению СД [24, 25]. Европейские и Американские рекомендации по лечению пациентов с СН и СД2 также обновили позиции иНГЛТ-2 в рекомендациях по лечению пациентов [26].

В августе 2019 г. Европейское общество кардиологов в сотрудничестве с EASD также обновило свои рекомендации по лечению пациентов СС3 и диабетом или предиабетом [26], где рекомендовало применение данной группы препаратов в качестве первой линии терапии в пациентов с высоким, очень высоким риском, а также у пациентов с документированным СС3, впервые отдав предпочтение иНГЛТ-2, а не метформину, для пациентов, ранее не получавших сахароснижающей терапии.

Данные обновления во многом связаны с результатами исследования DECLARE-TIMI 58, что открывает новые возможности раннего использования терапии, направленной на предупреждение и/или замедление прогрессирования нефропатии при СД2, снижение рисков развития СН при СД2, снижение СС-заболеваемости и смертности у пациентов с СД2, вне зависимости от наличия у них сердечно-сосудистой патологии в анамнезе, или имеющих множественные факторы риска CC3.

\section{ДОПОЛНИТЕЛЬНАЯ ИНФОРМАЦИЯ}

Источник финансирования. Обзор подготовлен при финансовой поддержке компании «АстраЗенека».

Конфликт интересов. Шестакова М.В. принимала участие в международных экспертных советах, связанных с проведением исследования, в качестве национального координатора исследования от России. Шестакова М.В. принимала участие в анализе и интерпретации данных, редактировании черновых вариантов рукописи и утверждала окончательную версию статьи для ее подачи к публикации.

Техническая поддержка в подготовке рукописи данной статьи выполнена группой MAG (Medical Advisors Group, http://mdwrt.com) в лице Якова Пахомова, Ирины Бодэ и Ильдара Хасанова. 


\section{СПИСОК ЛИТЕРАТУРЫ | REFERENCES}

1. Sulaiman MK. Diabetic nephropathy: recent advances in pathophysiology and challenges in dietary management. Diabetol Metab Syndr. 2019;11:7. doi: https://doi.org/10.1186/s13098-019-0403-4

2. Yuan CM, Nee R, Ceckowski KA, et al. Diabetic nephropathy as the cause of end-stage kidney disease reported on the medical evidence form CMS2728 at a single center. Clin Kidney J. 2017;10(2):257-262. doi: https://doi.org/10.1093/ckj/sfw112

3. Standl E. Heart failure in diabetes: from an increased risk to a treatment target. Diabetes Mellit. 2018;21 (5):399-403. doi: https://doi.org/10.14341/dm9846

4. Einarson TR, Acs A, Ludwig C, Panton UH. Prevalence of cardiovascular disease in type 2 diabetes: a systematic literature review of scientific evidence from across the world in 2007-2017. Cardiovasc Diabetol. 2018;17(1):83. doi: https://doi.org/10.1186/s12933-018-0728-6

5. Cavender MA, Steg PG, Smith SC Jr, et al. Impact of diabetes mellitus on hospitalization for heart failure, cardiovascular events, and death. Circulation. 2015;132(10):923-931. doi: https://doi.org/10.1161/CIRCULATIONAHA.114.014796

6. Dei Cas A, Khan SS, Butler J, et al. Impact of diabetes on epidemiology, treatment, and outcomes of patients with heart failure. JACC Hear Fail 2015;3(2):136-145 doi: https://doi.org/10.1016/j.jchf.2014.08.004

7. Wiviott SD, Raz I, Bonaca MP, et al. The design and rationale for the Dapagliflozin Effect on Cardiovascular Events (DECLARE)-TIMI 58 Trial. Am Heart J. 2018;200:83-89. doi: https://doi.org/10.1016/j.ahj.2018.01.012

8. Wiviott SD, Raz I, Bonaca MP, et al. Dapagliflozin and cardiovascular outcomes in type 2 diabetes. N Engl J Med. 2019;380(4):347-357. doi: https://doi.org/10.1056/NEJMoa1812389

9. Raz I, Mosenzon O, Bonaca MP, et al. DECLARE-TIMI 58: Participants' baseline characteristics. Diabetes Obes Metab. 2018;20(5):1102-1110. doi: https://doi.org/10.1111/dom.13217

10. Furtado RH, Bonaca MP, Raz I, et al. Dapagliflozin and cardiovascular outcomes in patients with type 2 diabetes mellitus and previous myocardial infarction. Circulation. 2019;139:2516-2527. doi: https://doi.org/10.1161/CIRCULATIONAHA.119.039996

11. Zinman B, Wanner C, Lachin JM, et al. Empagliflozin, cardiovascular outcomes, and mortality in type 2 diabetes. N Engl J Med. 2015;373(22):2117-2128. doi: https://doi.org/10.1056/NEJMoa1504720

12. Mosenzon O, Wiviott SD, Cahn A, et al. Effects of dapagliflozin on development and progression of kidney disease in patients with type 2 diabetes: an analysis from the DECLARE-TIMI 58 randomised trial. Lancet Diabetes Endocrinol. 2019;7(8):606-617. doi: https://doi.org/10.1016/S2213-8587(19)30180-9

13. Fitchett D, Zinman B, Wanner C, et al. Heart failure outcomes with empagliflozin in patients with type 2 diabetes at high cardiovascular risk: Results of the EMPAREG OUTCOME ${ }^{\oplus}$ trial. Eur Heart J. 2016;37(19):1526-1534. doi: https://doi.org/10.1093/eurheartj/ehv728

14. Wanner C, Inzucchi SE, Lachin JM, et al. Empagliflozin and progression of kidney disease in type 2 diabetes. N Eng/ J Med. 2016;375(4):323-334. doi: https://doi.org/10.1056/NEJMoa1515920

15. Neal B, Perkovic V, Mahaffey KW, et al.; CANVAS Programme
Collaborative Group. Canagliflozin and cardiovascular and renal events in type 2 diabetes. N Eng/ J Med. 2017;377(7):644-657. doi: https://doi.org/10.1056/NEJMoa1611925

16. Zelniker TA, Wiviott SD, Raz I, et al. SGLT2 inhibitors for primary and secondary prevention of cardiovascular and renal outcomes in type 2 diabetes: a systematic review and meta-analysis of cardiovascular outcome trials. Lancet. 2019;393(10166):31-39. doi: https://doi.org/10.1016/S0140-6736(18)32590-X

17. Verma S, Jüni P, Mazer CD. Pump, pipes, and filter: do SGLT2 inhibitors cover it all? Lancet. 2019;393(10166):3-5. doi: https://doi.org/10.1016/S0140-6736(18)32824-1

18. Zelniker TA, Braunwald E. Cardiac and renal effects of sodiumglucose co-transporter 2 inhibitors in diabetes: JACC stateof-the-art review. J Am Coll Cardiol. 2018;72(15):1845-1855. doi: https://doi.org/10.1016/j.jacc.2018.06.040

19. DVANCE Collaborative Group; Patel A, MacMahon S, Chalmers J, et al. Intensive blood glucose control and vascular outcomes in patients with type 2 diabetes. N Eng/ J Med. 2008;358(24):2560-2572. doi: https://doi.org/10.1056/NEJMoa0802987

20. Verma S, Jüni P, Mazer CD. Comment Pump, pipes, and filter: do SGLT2 inhibitors cover it all? Lancet. 2019;393(10166):3-5. doi: https://doi.org/10.1016/S0140-6736(18)32824-1

21. Zinman B, Inzucchi SE, Lachin JM, et al. Rationale, design, and baseline characteristics of a randomized, placebocontrolled cardiovascular outcome trial of empagliflozin (EMPA-REG OUTCOMETM). Cardiovasc Diabetol. 2014;13:102. doi: https://doi.org/10.1186/1475-2840-13-102

22. Kato ET, Silverman MG, Mosenzon O, et al. Effect of dapagliflozin on heart failure and mortality in type 2 diabetes mellitus. Circulation. 2019;139(22):2528-2536. doi: https://doi.org/10.1161/CIRCULATIONAHA.119.040130

23. Arnett DK, Blumenthal RS, Albert MA, et al. 2019 ACC/ AHA Guideline on the Primary Prevention of Cardiovascular Disease. J Am Coll Cardiol. 2019;74(10):e177-e232. doi: https://doi.org/10.1016/j.jacc.2019.03.010

24. Дедов И.И., Шестакова М.В., Майоров А.Ю., и др. Алгоритмы специализированной помощи больным сахарным диабетом под ред. И.И. Дедова, М.В. Шестаковой, А.Ю. Майорова. 9-й вып. // Сахарный диабет. - 2019. - № 22. - № S1-1. - С. 1-144. [Dedov II, Shestakova MV, Mayorov AYu, et al. Standards of specialized diabetes care. Ed. by I.I. Dedov, M.V. Shestakova, A.Yu. Mayorov. 9th ed. Diabetes Mellitus. 2019;22(S1-1):1-144. (In Russ.)]. doi: https://doi.org/10.14341/DM221S1

25. Dunlay SM, Givertz MM, Aguilar D, et al.; American Heart Association Heart Failure and Transplantation Committee of the Council on Clinical Cardiology; Council on Cardiovascular and Stroke Nursing; and the Heart Failure Society of America. Type 2 diabetes mellitus and heart failure: a scientific statement from the American Heart Association and the Heart Failure Society of America: this statement does not represent an update of the 2017 ACC/AHA/HFSA heart failure guideline update. Circulation. 2019;140(7):e294-e324. doi: https://doi.org/10.1161/CIR.0000000000000691.

26. Cosentino F, Grant PJ, Aboyans V, et al. 2019 ESC Guidelines on diabetes, pre-diabetes, and cardiovascular diseases developed in collaboration with the EASD. Eur Heart J. 2020;41 (2):255-323. doi: https://doi.org/10.1093/eurheartj/ehz486

\section{ИНФОРМАЦИЯ ОБ АВТОРАХ [AUTHORS INFO]}

Шестакова Марина Владимировна, д.м.н., профессор, академик РАH [Marina V. Shestakova, MD, PhD, Professor]; адрес: Россия, 117036, Москва, ул. Дм. Ульянова, д. 11 [address: 11 Dm.Ulyanova street, 117036 Moscow, Russia]; ORCID: https://orcid.org/0000-0002-5057-127X; eLibrary SPIN: 7584-7015; e-mail: nephro@endocrincentr.ru

\section{ЦИТИРОВАТЬ:}

Шестакова M.В. Исследование DECLARE-TIMI 58 в контексте EMPA-REG OUTCOME и CANVAS // Caхарный диабет. — 2019. — T. 22. — №6. - C. 592-601. doi: 10.14341/DM10289

\section{TO CITE THIS ARTICLE:}

Shestakova MV. DECLARE-TIMI 58 trial in the context of EMPA-REG OUTCOME and CANVAS. Diabetes Mellitus. 2019;22(6):592-601. doi: 10.14341/DM10289 